\title{
Gyroresonant interactions between the radiation belt electrons and whistler mode chorus waves in the radiation environments of Earth, Jupiter, and Saturn: A comparative study
}

\author{
Y. Y. Shprits, ${ }^{1,2}$ J. D. Menietti, ${ }^{3}$ X. Gu, ${ }^{2}$ K. C. Kim, ${ }^{4}$ and R. B. Horne ${ }^{5}$ \\ Received 26 June 2012; revised 16 August 2012; accepted 20 September 2012; published 21 November 2012.
}

[1] In the current study we perform a comparative analysis of the gyroresonant interactions of whistler mode waves with radiation belt electrons in the magnetospheres of Earth, Jupiter, and Saturn. Our primary goal is to evaluate the effect of resonant wave-particle interactions with chorus waves and determine whether chorus waves can produce net acceleration or net loss of radiation belt electrons on the outer planets. The ratio of plasma frequency to gyrofrequency is a key parameter that determines the efficiency of the pitch angle and energy resonant scattering. We present a comparison of statistical maps of the ratio of plasma frequency to gyrofrequency for Jupiter, Saturn and Earth in terms of radial distance and latitude. Preliminary maps of the plasma frequency to gyrofrequency ratio and 2D simulations of pitch angle and energy diffusion using the Versatile Electron Radiation Belt (VERB) indicate that the Kronian plasma environment is not likely to support as efficient gyroresonant interactions with whistler mode chorus waves as in the Terrestrial or Jovian environments. Inefficiency of the local acceleration by whistler mode waves in the Kronian environment raises important questions about the origin of the relativistic electrons in the Saturn's radiation belts. Two-dimensional diffusive simulations of local acceleration and loss to the atmosphere using the VERB code confirm previous suggestions that the acceleration of electrons may be very efficient in the outer radiation belt of Jupiter. However, sensitivity simulations also show that the result of the competition between acceleration and loss in the Jupiter's magnetosphere strongly depends on the currently unknown latitudinal distribution of chorus waves that will be provided by the upcoming Juno mission. If waves extend to high latitudes, it is likely that the loss rates due to whistler mode waves will exceed energization rates.

Citation: Shprits, Y. Y., J. D. Menietti, X. Gu, K. C. Kim, and R. B. Horne (2012), Gyroresonant interactions between the radiation belt electrons and whistler mode chorus waves in the radiation environments of Earth, Jupiter, and Saturn: A comparative study, J. Geophys. Res., 117, A11216, doi:10.1029/2012JA018031.

\section{Introduction}

[2] The first observations of the decimeter emissions from Jupiter provided evidence of the presence of trapped radiation in Jupiter's magnetosphere [Drake and Hvatum, 1959; Radhakrishnan and Roberts, 1960]. Later, remote sensing of the Jovian magnetosphere based on the measurements of the

\footnotetext{
${ }^{1}$ Skolkovo Institute of Science and Technology, Moscow, Russia.

${ }^{2}$ Institute of Geophysics and Planetary Physics and Department of Earth and Space Sciences, University of California, Los Angeles, California, USA.

${ }^{3}$ Department of Physics and Astronomy, University of Iowa, Iowa City, Iowa, USA.

${ }^{4}$ Korea Astronomy and Space Science Institute, Daejeon, South Korea.

${ }^{5}$ British Antarctic Survey, Cambridge, UK.

Corresponding author: Y. Y. Shprits, Institute of Geophysics and Planetary Physics and Department of Earth and Space Sciences, University of California, 405 Hilgard Ave., Box 951565, 7127 Math Sciences Bldg., Los Angeles, CA 90095-1565, USA. (yshprits@atmos.ucla.edu)

(C2012. American Geophysical Union. All Rights Reserved. 0148-0227/12/2012JA018031
}

synchrotron radiation [e.g., Branson, 1968; Stannard and Conway, 1976; Bolton et al., 2002] provided detailed maps and information on pitch angle distributions of the ultrarelativistic electron population in the Jovian magnetosphere. The highest intensity of the synchrotron radiation was observed around $1.4 \mathrm{R}_{\mathrm{J}}$ [Drake and Hvatum, 1959; Carr and Gulkis, 1969]. In situ measurements also showed the presence of $>1 \mathrm{MeV}$ protons as well as oxygen, sulfur and sodium ions [Cohen et al., 2001].

[3] Whether Saturn's environment has been populated by trapped energetic particles or not remained unknown until the first measurements by Pioneer 11 [McKibben and Simpson, 1980; Simpson et al., 1980; Van Allen et al., 1980]. Voyager and Cassini provided the first detailed measurements of the Kronian radiation environment.

[4] Modeling of the inner part of the trapped radiation of Jupiter [Santos-Costa and Bourdarie, 2001; Santos-Costa et al., 2008] and Saturn [Santos-Costa et al., 2003] included radial diffusion, interactions with neutrals, and synchrotron radiation loss which is most important for Jupiter's inner 
region. Santos-Costa et al., [2003] modeled the Saturn's radiation belts by including a number of competing acceleration and loss processes. The source of electrons was provided by the boundary condition at $\mathrm{L}=6$ and radial transport was modeled by a radial diffusion with empirically selected parameters for the radial diffusion. The radial diffusion coefficient was chosen to be in the form $D_{L L}=D_{0} L^{n}$ where n was assumed to be equal to 3 and $D_{0}$ equal to $10^{-8} \mathrm{~s}^{-1}$. While the results of simulations were very similar to observations in the inner region, authors noted that "Wave particle interaction effects have not been introduced in our model." They also noted that detailed study of wave-particle interactions should be a subject of future studies. Similar approach was used by Sicard and Bourdarie [2004] who modeled Jupiter's radiation belts by setting up the outer boundary of 3D simulations at $\mathrm{L}=9.5$ and Sicard et al. [2004] with the boundary at $\mathrm{L}=6$. Most recently, Lorenzato et al. [2012] compared the 3D global modeling of Saturn's radiation belts with observations from various spacecraft. For the first time authors included wave-particle interactions. However, the detailed study of the sensitivity of simulations to the assumed parameters, mixed term scattering, and comparison of the waveparticle interactions on Saturn and other planets was beyond the scope of the study.

[5] The Earth's radiation belts have received substantial attention in recent years. Relativistic electrons in the Earth's radiation belts can penetrate the protective shielding surrounding satellites and can be hazardous to the satellite's electronics [e.g., Baker, 2002]. It is now widely accepted that the presence of radiation belt electrons in the terrestrial environment results from radial diffusion [e.g., Kellogg, 1959; Fälthammar, 1965, Tverskoy, 1965; Shprits and Thorne, 2004] and also local acceleration [e.g., Summers et al., 1998; Horne and Thorne, 1998; Horne et al., 2005a; Shprits et al., 2006a; Miyoshi et al., 2003]. A number of multidimensional codes that combine radial diffusion and local processes due to gyroresonant scattering [e.g., Varotsou et al., 2005; Shprits et al., 2009; Albert et al., 2009; Su et al., 2010] have recently been developed and validated.

[6] Unlike the Earth's magnetosphere, which is strongly driven by solar wind, the dynamic evolution of plasma populations in the planetary magnetospheres is primarily determined by internal processes. However, there are a number of similarities between the physical processes that affect the most energetic populations of the terrestrial and planetary magnetospheres. Inward radial diffusion driven by the Ultra-Low Frequency (ULF) waves provides radial transport of particles and may result in acceleration of particles in the Earth's radiation belts [Kellogg, 1959]. Similar inward transport processes which include radial diffusion induced by electrostatic fluctuations and centrifugally driven instabilities in the magnetospheres of Jupiter and Saturn, can provide a net diffusive transport from the outer into the inner planetary magnetospheres [Brice and McDonough, 1973; Siscoe and Summers, 1981; Kivelson et al., 1997].

[7] Horne et al. [2008] showed that, similar to the Earth's radiation belts, relativistic electrons in the Jovian radiation belts can be accelerated locally by chorus waves that take free energy for the excitation and growth form few $\mathrm{keV}$ electrons [Katoh et al., 2011]. In particular, Horne et al. [2008] noticed that the local acceleration by chorus waves on Jupiter may serve as the processes of providing the seed population for further acceleration to ultra-relativistic energies in region around $10 \mathrm{R}_{\mathrm{J}}$. In the described process of excitation of waves and local acceleration, waves allow the transfer of energy from the low-energy population of electrons to the high-energy population.

[8] In the current study, we perform a comparative analysis of the conditions for the local acceleration of radiation belt electrons in the magnetospheres of Earth, Jupiter, and Saturn. The competition between the local acceleration and loss to the atmosphere can be described by the modified Fokker-Planck equation, which can be written as [i.e., Schulz and Lanzerotti, 1974; Shprits et al., 2008]:

$$
\begin{aligned}
\frac{\partial f}{\partial t}= & \left.\frac{1}{p^{2}} \frac{\partial}{\partial p}\right|_{\alpha_{0}, L} p^{2}\left(\left.\left\langle D_{p p}\left(\alpha_{0}, p\right)\right\rangle \frac{\partial f}{\partial p}\right|_{\alpha_{0}, L}+\left.\left\langle D_{p \alpha_{0}}\left(\alpha_{0}, p\right)\right\rangle \frac{\partial f}{\partial \alpha_{0}}\right|_{p, L}\right) \\
& +\left.\frac{1}{T\left(\alpha_{0}\right) \sin \left(2 \alpha_{0}\right)} \frac{\partial}{\partial \alpha_{0}}\right|_{p, L} T\left(\alpha_{0}\right) \sin \left(2 \alpha_{0}\right) \\
& \cdot\left(\left.\left\langle D_{\alpha_{0} \alpha_{0}}\left(\alpha_{0}, p\right)\right\rangle \frac{\partial f}{\partial a_{0}}\right|_{p, L}+\left.\left\langle D_{\alpha_{0} p}\left(\alpha_{0}, p\right)\right\rangle \frac{\partial f}{\partial p}\right|_{\alpha_{0}, L}\right)-\frac{f}{\tau}, \quad(1)
\end{aligned}
$$

where $f$ is the Phase Space Density (PSD), $t$ is the time, $p$ is the relativistic momentum, $L$ is the $L^{*}$ parameter, and $\alpha_{0}$ is the equatorial pitch angle. Relativistic momentum $p$ can be related to the kinetic energy as $E=\sqrt{p^{2} c^{2}+m^{2} c^{4}}-m c^{2}$ and the momentum diffusion is closely related to energy diffusion. $\left\langle D_{p p}\right\rangle,\left\langle D_{\alpha_{0} \alpha_{0}}\right\rangle$, and $\left\langle D_{p \alpha_{0}}\right\rangle\left(\equiv\left\langle D_{\alpha_{0} p}\right\rangle\right)$ are the momentum, pitch angle, and mixed bounce and drift-averaged diffusion coefficients, $\tau$ is the electron lifetime, which we assume to be equal to a quarter of the bounce time inside the loss cone and to be infinite outside the loss cone. $T\left(\alpha_{0}\right)$ is a function, related to bounce motion, and in a dipolar field is approximated following [Lenchek et al., 1961]:

$$
\mathrm{T}\left(\alpha_{0}\right)=1.3802-0.3198\left(\sin \alpha_{0}+\sin ^{1 / 2} \alpha_{0}\right) .
$$

\section{Latitudinal and Radial Distribution of the Plasma Frequency to Gyrofrequency Ratio}

\subsection{Jovian Cold Plasma Environment}

[9] The efficiency of wave-particle interactions strongly depends on the value of the plasma frequency to gyrofrequency ratio $\omega_{p e} /\left|\Omega_{c e}\right|$ [e.g., Horne et al. 2005b]. To compare the environments of Earth, Saturn and Jupiter, we first compare the ratio of the plasma frequency to gyrofrequency for these planets, which depends on the strength of the local background magnetic field and plasma density. A similar map for Jupiter has been recently shown in Katoh et al. [2011].

[10] Based on the observations of the Plasma Particle Investigation (PLS) on board the Galileo spacecraft in Jupiter's magnetosphere during the period of May 4 through June 22, 1997, Frank et al. [2002] derived a parameterization of the near equatorial electron density inside $20 R_{J}$,

$$
N_{e q}(L)=3.2 \times 10^{8} \mathrm{~L}^{-6.9} \mathrm{~cm}^{-3},
$$

where $L$ is the approximate distance between the center of Jupiter and the field line in the equatorial plane. This model has been validated by Bagenal and Delamere [2011], who 

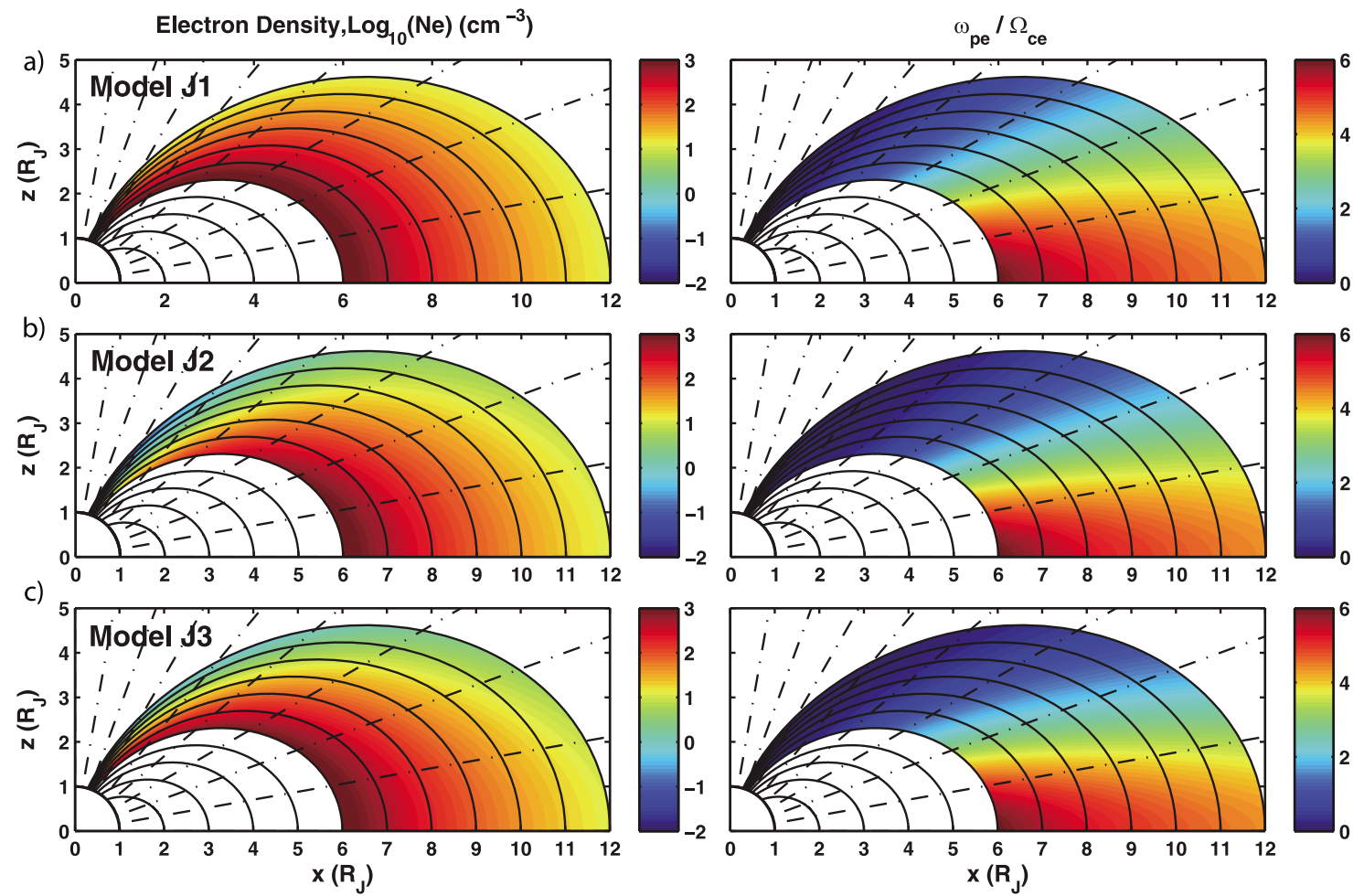

Figure 1. Modeled electron density $\left(\log _{10}(\mathrm{Ne}), \mathrm{cm}^{-3}\right)$ and $\omega_{p e} / \Omega_{c e} \mid$ distribution in the inner magnetosphere of Jupiter. (left) The density for (a) model J1, (b) model J2, and (c) model J3. (right) The corresponding ratios of $\omega_{p e} /\left|\Omega_{c e}\right|$. The solid line represents the L-shell and the dash-dotted line represents the latitude from $0^{\circ}$ to $90^{\circ}$ with a $10^{\circ}$ interval.

combined measurements from Voyager and Galileo and derived a profile of equatorial plasma density between the orbit of Io and $10 R_{J}$.

[11] Since the dependence of electron density on latitude $\lambda$ is not well known, we adopt three different models in this study, herein referred to as $\mathrm{J} 1, \mathrm{~J} 2$, and $\mathrm{J} 3$, to study the sensitivity of our assumptions to the latitudinal distribution of density. For model J1, we assume that density does not change with latitude.

$$
N(L, \lambda)=N_{e q}(L)(\text { Model J1) }
$$

while for model $\mathrm{J} 2$, the density along the field line is inversely proportional to the magnetic field strength

$$
N(L, \lambda)=N_{e q}(L)\left[\left(1+3 \sin ^{2} \lambda\right)^{1 / 2} / \cos ^{6} \lambda\right]^{-1}(\text { Model J2), }
$$

[12] The third model is adopted following Persoon et al. [2006]

$$
N(L, \lambda)=N_{e q}(L)\left(-L\left(1-\cos ^{6} \lambda\right)\right)^{1 / 2} / 3 H^{2}(\text { Model J3 }),
$$

where $H$ is the scale height and given by Bagenal and Delamere [2011] as below, $H=10^{a_{1}+a_{2} r+a_{3} r^{2}+a_{4} r^{3}+a_{5} r^{4}}$, with $r=\log _{10}(L)$ and a set of parameters $a_{1}=-0.116, a_{2}=$ 2.14, $a_{3}=-2.05, a_{4}=0.491$, and $a_{5}=0.126$.

[13] The distribution of the plasma frequency to gyrofrequency ratio is on the scale of 2 to 6 for a wide range of L-shells and latitudes, creating preferential conditions for the local acceleration of electrons [e.g., Horne and Thorne, 1998; Horne et al., 2005b].

\subsection{Kronian Cold Plasma Environment}

[14] Based on Radio and Plasma Wave Science (RPWS) measurements taken by Cassini, Persoon et al. [2006] developed a diffusive equilibrium model for the distribution of water group ions, hydrogen ions, and electrons in the inner region of Saturn's magnetosphere. Furthermore, they derived the electron density in Saturn's magnetosphere from the charge neutrality condition, which we denote as model S1 (Figure 2a).

[15] We also present another density model for Saturn (model S2), using the Thomsen et al. [2010] equatorial density model

$$
N_{e q}(L)=A \exp (-B L)
$$

where $A=627$ and $B=0.517$, and a latitudinal dependence given by equation (6) with $H=\sqrt{L^{2} / 3 C}, C=4.56$.

[16] Both models show a relatively high ratio of plasma frequency to gyrofrequency of above 10 for L-shells greater than 5. A comparison of Figures 1 and 2 shows that the cold electron plasma environment on Jupiter provides preferential conditions for local acceleration by whistler mode waves while the high ratio of plasma frequency to gyrofrequency in Saturn's inner magnetosphere does not allow for such efficient exchange of energy between the electrons and waves. Other processes such as inward radial diffusion and possibly interactions with other plasma waves may play important 

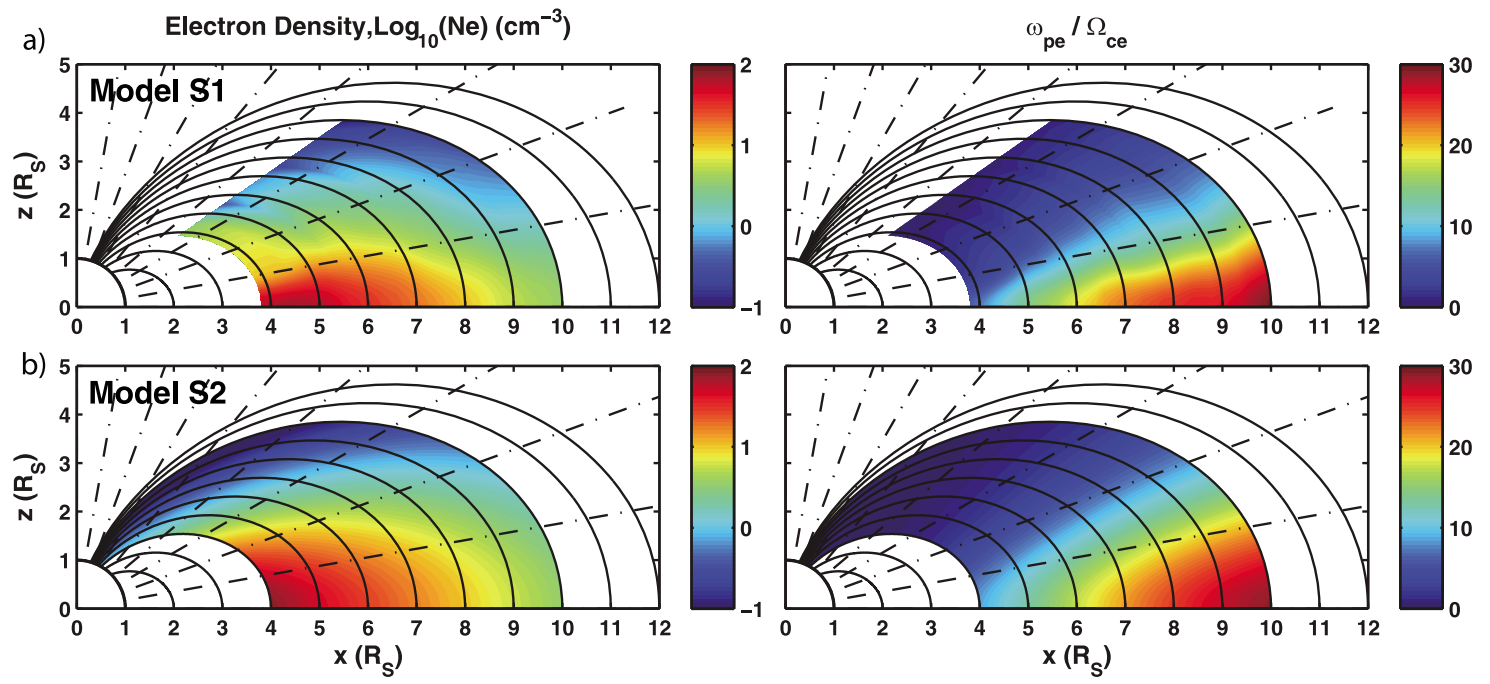

Figure 2. Model electron density $\left(\log _{10}(\mathrm{Ne}), \mathrm{cm}^{-3}\right)$ and $\omega_{p e} / \Omega_{c e} \mid$ distribution in the inner magnetosphere of Saturn. (left) (a) Model S1 and (b) model S2 and (right) the corresponding values for $\omega_{p e} /\left|\Omega_{c e}\right|$.

roles in the acceleration of electrons to relativistic energies in the Kronian magnetosphere.

[17] A comparison of plasma environments for all three planets (Figure 3) shows that, while local acceleration in the Earth's magnetosphere can occur for the relatively narrow range of L-shells between the plasmapause, where density drops to low values to approximately $L=7$, which is close to the boundary of trapping, the magnetosphere of Jupiter can allow for local acceleration for a wide range of L-shells. Unlike the Jovian magnetosphere, Saturn's magnetosphere does not provide preferential conditions for the efficient local acceleration by chorus waves in the region outside of
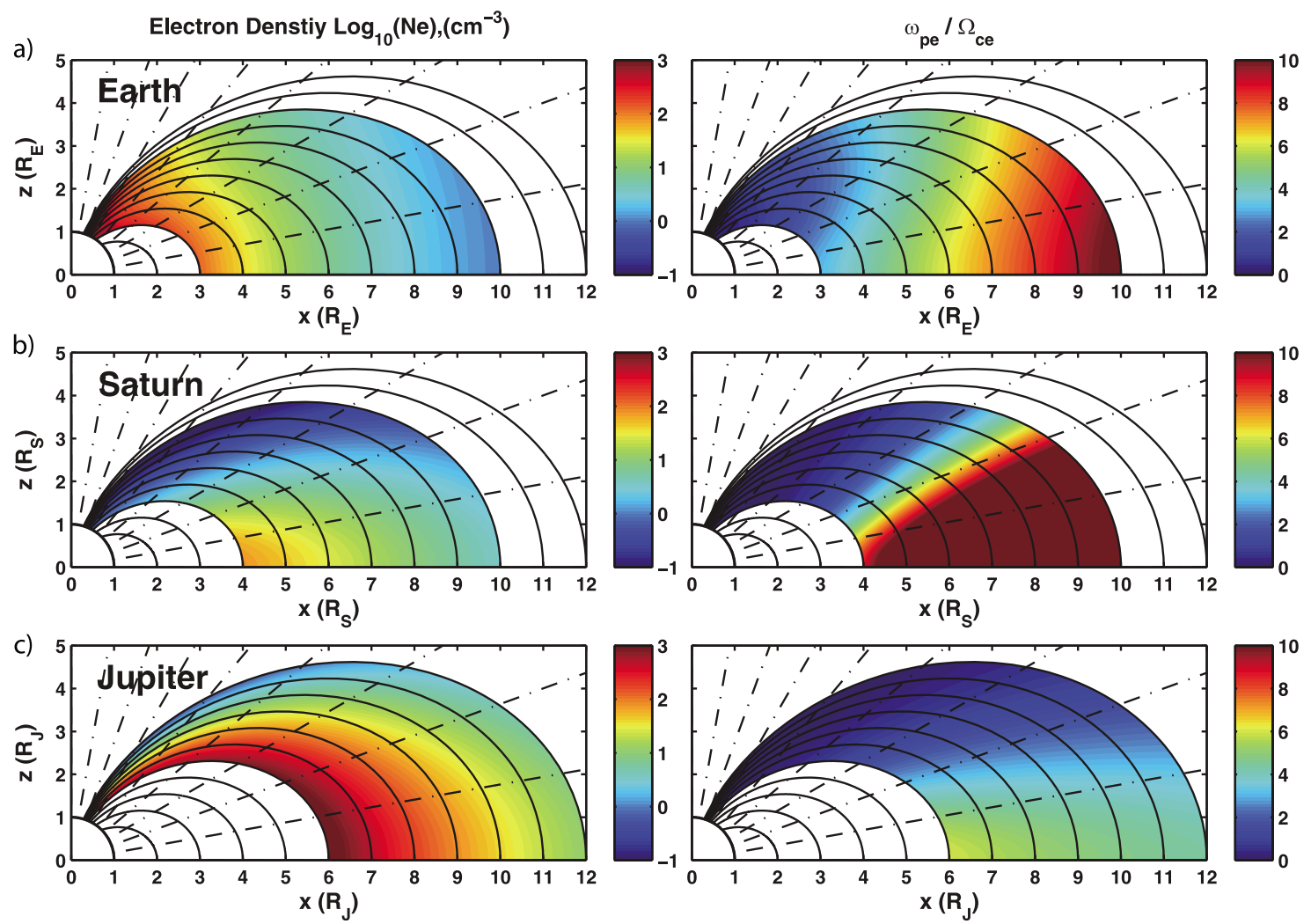

Figure 3. Model electron density $\left(\log _{10}(\mathrm{Ne}), \mathrm{cm}^{-3}\right)$ and $\omega_{p e} /\left|\Omega_{c e}\right|$ distribution in the inner magnetosphere of Earth, Saturn, and Jupiter. (a) The density model given by Sheeley et al. [2001] for Earth, (b) model S2 in Figure 2 for Saturn, and (c) model J3 in Figure 1 for Jupiter. 
6 Rs, where losses due to the interactions with dusty plasmas are not significant.

\section{Chorus Waves in the Magnetospheres of Saturn and Jupiter}

[18] Whistler mode chorus emissions are often observed in the Earth's magnetosphere as the rising tones above and below one half of the electron gyrofrequency [Tsurutani and Smith, 1977; Koons and Roeder, 1990] in the regions of low plasma density. Chorus waves in the Earth's magnetosphere are confined to approximately $15^{\circ}$ of the geomagnetic equator on the night side [e.g., Meredith et al., 2003] and up to $40^{\circ}-45^{\circ}$ on the day side [Bunch et al., 2011, 2012]. The total amplitudes of typical chorus waves in the Earth environment vary from 10 to $100 \mathrm{pT}$ and are strongly correlated with geomagnetic activity [Meredith et al., 2003; Shprits et al., 2007].

[19] The rapid rotation of Jupiter and Saturn drives the interchange instability between the cold and dense plasma in the inner region and the hot and tenuous plasma from the outer region. Interchange instability results in a hotter inflow plasma that forms a temperature anisotropy and excites whistler mode waves [Bolton et al., 1997; Thorne et al., 1997; Rymer et al., 2008; Liu et al., 2010]. The first observations of chorus waves in the Kronian magnetosphere were done by the Voyager spacecraft [Gurnett et al., 1981; Scarf et al. 1982]. Spectrograms from Voyager showed rising frequency elements characteristic of chorus that looked similar to Earth's emissions near the 1/2 gyrofrequency. The Cassini mission, with its complex of radio receivers, has greatly expanded our knowledge of chorus emission from Saturn [e.g., Hospodarsky et al., 2008]. Figure 4a shows an example of a frequency-time spectrogram for day 352 of 2008, with the magnetic field spectral density color-coded. The data were obtained as the spacecraft crossed the magnetic equator from north to south. Chorus emissions in Jupiter's magnetosphere were first detected by Voyagers 1 and 2 [e.g., Scarf and Gurnett, 1977]. Figure 4b shows a typical example of chorus emissions observed by the Galileo spacecraft. The dynamic spectrogram shows waves in the frequency range from $300 \mathrm{~Hz}$ to $20 \mathrm{kHz}$. A number of constant-frequency interference lines are present for $f>5 \mathrm{kHz}$. Chorus emission is seen in the frequency range from a few hundred $\mathrm{Hz}$ up to, but seldom exceeding, about $f_{c e} / 2$. The frequency of the chorus band peaks every time the satellite crosses the magnetic equator, forming a repeating pattern. Note that the waves remain strong as the satellite moves to higher magnetic latitudes.

[20] The dynamic spectrograms (Figure 4) clearly show that waves are commonly present below the $1 / 2$ gyrofrequency for the magnetospheres of both Jupiter and Saturn and have wave amplitudes that are similar to those of the waves observed on the Earth. A detailed statistical study of the wave environments of Jupiter and Saturn is beyond the scope of this initial study and will be a subject of future studies.

\section{Resonant Wave Particle Interactions}

[21] Based on the observations from Voyager and Cassini, Figure 4, and the typical properties of the Earth's chorus waves [e.g., Li et al., 2007], we adopt a model for the distribution of the plasma waves, which is summarized in Table 1 . Since the latitudinal distribution of chorus waves in the Kronian and Jovian magnetospheres is not known, we first assume that waves are confined to within 10 degrees of the magnetic equator, following Horne et al. [2008]. Waves are assumed to be present at all MLT at all times for both Jupiter's and Saturn's magnetospheres (J. D. Menietti, et al., Chorus emissions observed at Jupiter and Saturn and possible electron acceleration, manuscript in preparation, 2012.).

[22] Figure 5 shows the results of calculations of the bounce-averaged diffusion coefficients with the Full Diffusion Code (FDC) [Ni et al., 2008; Shprits and Ni, 2009], accounting for Landau $(n=0)$ and higher order $(n \leq 5)$ resonant scattering. The code can be run on multiple processors in the UCLA computer cluster. The code uses a formulation of Glauert and Horne [2005] and Albert [2005]. We assume that the wave power spectral density $B^{2}(\omega)$ is distributed according to a Gaussian frequency distribution with fixed cut-offs $\omega_{l c}$ and $\omega_{u c}$, median value $\omega_{m}$, bandwidth $d \omega$, and $B_{w}$ averaged wave amplitude. We also assume that the wave normal distribution is Gaussian over $X$, where $X=\tan (\theta)$, $X_{m}=\tan \left(\theta_{m}\right), X_{w}=\tan (\mathrm{d} \theta), \theta$ is the wave normal angle, $\theta_{m}$ is the peak wave normal angle, and $d \theta$ is a parameter describing the angular width of the distribution.

[23] While pitch angle scattering results in the loss of particles from the system, momentum diffusion, which is often referred to as energy diffusion, results in the acceleration of particles. The net effect of wave-particle interactions depends on a number of factors including the ratio of plasma frequency to gyrofrequency, the amplitudes of waves, the spectral properties of waves, and on the distribution in the wave normal angle and the latitudinal extent of waves [e.g., Horne et al., 2005b; Thorne et al., 2005; Shprits et al., 2006b].

[24] The pitch angle scattering rates for all three planets are higher than the energy scattering rates. However, that does not necessarily mean that losses will dominate acceleration [Shprits et al., 2009]. While loss rates are primarily determined by the value near the edge of the loss cone, where pitch angle scattering rates are often at a minimum [Roberts, 1969; Shprits et al., 2006d; Albert and Shprits, 2009], acceleration will occur at all pitch angles and may overtake losses [e.g., Li et al., 2007]. Mixed diffusion terms can also affect evolution of the phase space density and usually act to inhibit local accelerations [Albert and Young, 2005; Tao et al., 2009; Subbotin et al., 2011]. To quantitatively study the effect of pitch angle and energy scattering by chorus waves, in section 5 we present $2 \mathrm{D}$ simulations with the $2 \mathrm{D}$ version of the Versatile Electron Radiation Belt (VERB) diffusion code [Shprits et al., 2006a, 2009; Subbotin and Shprits, 2009].

\section{The 2-D Simulations of the Pitch Angle, Energy, and Mixed Scattering by Chorus Waves}

[25] We first perform simulations of the pitch angle and energy scattering using an analytical spectrum given by Albert and Young [2005] as an initial condition

$$
\mathrm{J}=\mathrm{e}^{-(\mathrm{E}-0.2) / 0.1}\left(\sin \alpha-\sin \alpha_{\text {loss cone }}\right),
$$

where $E$ is the kinetic energy in $\mathrm{MeV}$ and $\alpha$ is an equatorial pitch angle. The boundary conditions are summarized in Table 2. 
a)

\section{Saturn Wave Spectral Denstiy}
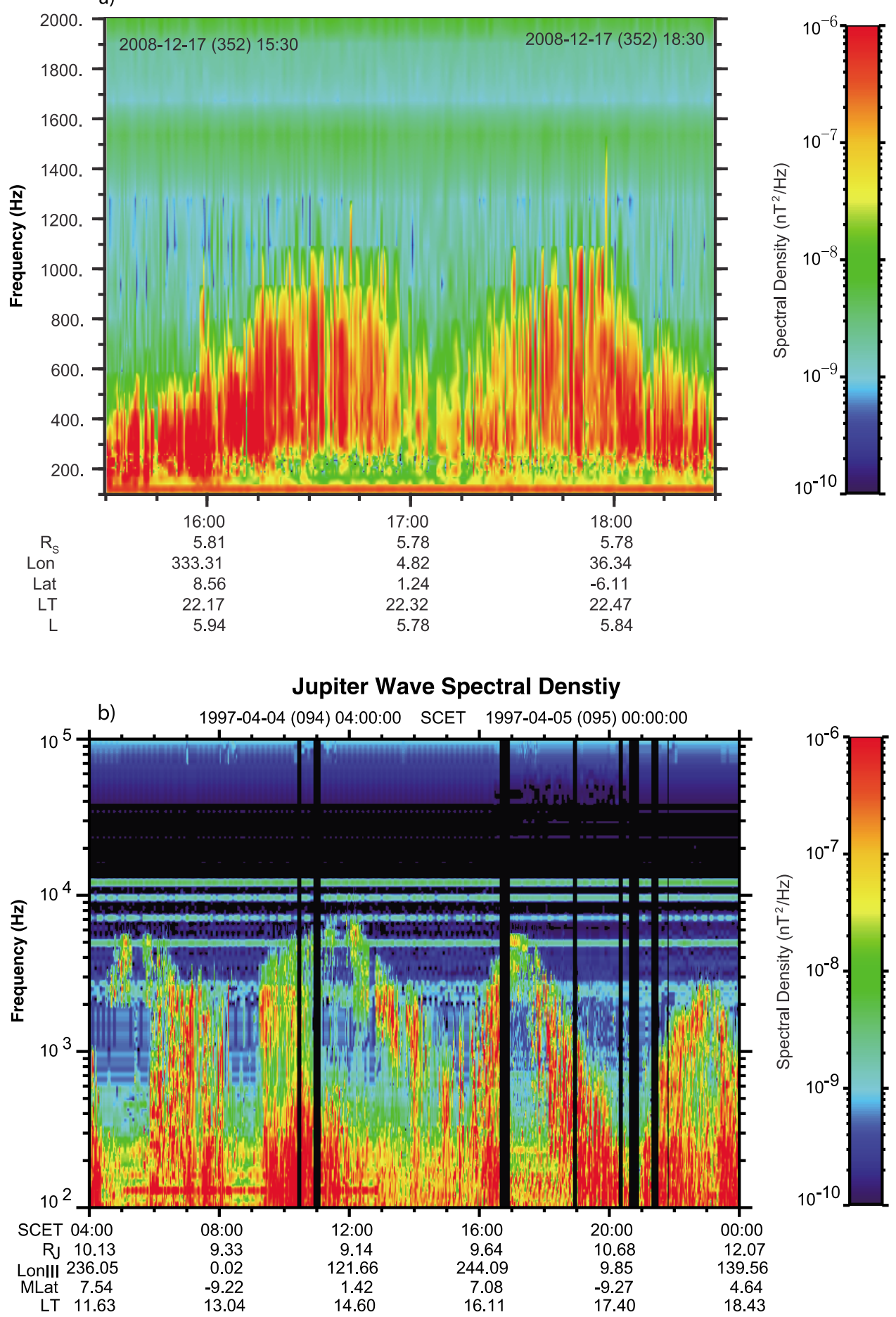

Figure 4. Dynamic spectrograms obtained from observations of chorus waves on (a) Saturn and (b) Jupiter. The wave intensity is plotted as a function of spacecraft event time (SCET), system 3 longitude (Lon ${ }_{\text {III }}$ ), magnetic latitude (Mlat), and local time (LT).

[26] Figure 6 shows a comparison of the 2D simulations that include pitch angle and energy diffusion for the local scattering of electrons in Jupiter's and Saturn's radiation belts. Results for Saturn's radiation belt are given at $\mathrm{L}=6$, in the region where plasma frequency to gyrofrequency is relatively low, to give local acceleration a fair chance. At higher L-shells (not shown in this manuscript), acceleration at high energies is less efficient. Following Horne et al. [2008], we perform simulations for Jupiter at $L=10$, which is a region where plasma frequency to gyrofrequency is relatively low. The ratio of plasma frequency to gyrofrequency 
Table 1. Assumed Wave Properties

\begin{tabular}{|c|c|c|c|c|c|}
\hline Planet & $\begin{array}{c}\text { Wave Intensity } \\
B_{\mathrm{w}}(\mathrm{pT})\end{array}$ & $\lambda_{\max }$ & $\begin{array}{l}\text { MLT Distribution } \\
\text { of Wave Power (\%) }\end{array}$ & $\begin{array}{c}\text { Wave Spectral } \\
\text { Properties }\end{array}$ & $\begin{array}{c}\text { Wave Propagation } \\
\text { Angle }\end{array}$ \\
\hline Jupiter and Saturn & 30 & $10^{\circ}$ & 100 & $\begin{array}{l}\omega_{\mathrm{m}} / \Omega_{\mathrm{e}}=0.1 \\
\delta \omega / \Omega_{\mathrm{e}}=0.05 \\
\omega_{\mathrm{lc}} / \Omega_{\mathrm{e}}=0.05 \\
\omega_{\mathrm{uc}} / \Omega_{\mathrm{e}}=0.25\end{array}$ & $\begin{array}{c}\theta_{\mathrm{m}}=0^{\circ} \\
\delta \theta=30^{\circ} \\
\theta_{\mathrm{lc}}=0^{\circ} \\
\theta_{\mathrm{uc}}=70^{\circ}\end{array}$ \\
\hline Earth Chorus Day & 30 & $35^{\circ}$ & 25 & $\begin{array}{l}\omega_{\mathrm{m}} / \Omega_{\mathrm{e}}=0.2 \\
\delta \omega / \Omega_{\mathrm{e}}=0.1 \\
\omega_{\mathrm{lc}} / \Omega_{\mathrm{e}}=0.1 \\
\omega_{\mathrm{uc}} / \Omega_{\mathrm{e}}=0.3\end{array}$ & $\begin{array}{c}\theta_{\mathrm{m}}=0^{\circ} \\
\delta \theta=30^{\circ} \\
\theta_{\mathrm{lc}}=0^{\circ} \\
\theta_{\mathrm{uc}}=45^{\circ}\end{array}$ \\
\hline Earth Chorus Night & 30 & $15^{\circ}$ & 25 & $\begin{array}{l}\omega_{\mathrm{m}} / \Omega_{\mathrm{e}}=0.35 \\
\delta \omega / \Omega_{\mathrm{e}}=0.15 \\
\omega_{\mathrm{lc}} / \Omega_{\mathrm{e}}=0.05 \\
\omega_{\mathrm{uc}} / \Omega_{\mathrm{e}}=0.65\end{array}$ & $\begin{array}{c}\theta_{\mathrm{m}}=0^{\circ} \\
\delta \theta=30^{\circ} \\
\theta_{\mathrm{lc}}=0^{\circ} \\
\theta_{\mathrm{uc}}=45^{\circ}\end{array}$ \\
\hline
\end{tabular}

$\omega_{p e} / \Omega_{c e}$ at Jupiter is calculated to be 4.8 at the equator and decreases with latitude. This value is very similar to the value of $\omega_{p e} /\left|\Omega_{c e}\right|$ in the heart of the Earth's radiation belts at $\mathrm{L}=4.5$. The value of $\omega_{p e} / \Omega_{c e} \mid$ on Saturn is much higher and is approximately 17.5 at the Equator at $\mathrm{L}=6$.

[27] While pitch angle diffusion alone is not able to produce a significant loss of electrons on the scale of a few days for both Jupiter and Saturn, it changes the shape of the distribution function making it more isotropic than the initial sine distribution function. The changes in the pitch angle distribution are more pronounced for the Jupiter radiation belts due to the lower plasma frequency to gyrofrequency as discussed above.

[28] When energy diffusion is included, local acceleration dominates over pitch angle scattering for high-energy electrons for both of the planets, and acceleration is clearly much more efficient for Jupiter. The addition of mixed diffusion only slightly changes the pitch angle distribution, and acts to suppress the local acceleration. The effect of mixed diffusion is most pronounced at $500 \mathrm{keV}$ and small pitch-angels,
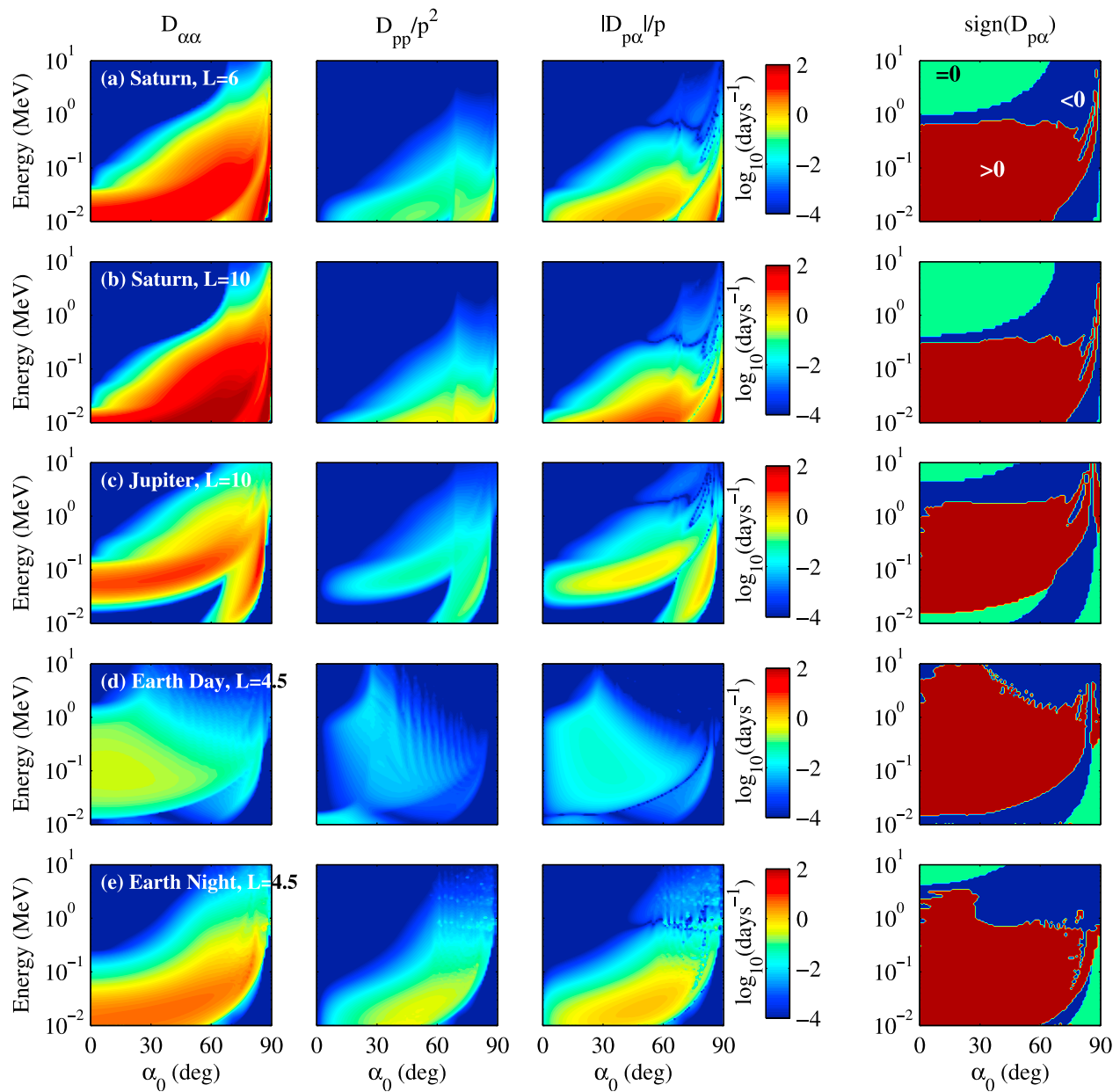

Figure 5. Pitch angle, energy, and mixed scattering rates: (a) Saturn, $L=6$, (b) Saturn, $L=10$, (c) Jupiter $L=10$, (d) Earth day side $L=4.5$, (e) Earth Night side $L=4.5$. 
Table 2. Boundary Conditions Used for the 2-D VERB Simulations

\begin{tabular}{lcc}
\hline Boundary & Condition & Physical Approximation \\
\hline$\alpha=0.3^{\circ}$ & $f=0$ & $\begin{array}{c}\text { Empty loss cone in the weak } \\
\text { diffusion regime }\end{array}$ \\
$\alpha=89.7^{\circ}$ & $\mathrm{d} f / \mathrm{d} \alpha=0$ & $\begin{array}{c}\text { Flat pitch angle distribution at } 90^{\circ} \\
\text { Balance of convective sources } \\
\text { and losses }\end{array}$ \\
$\mathrm{E}_{\min }=100 \mathrm{keV}$ & $f=$ constant & $\begin{array}{c}\text { Absence of higher energy electrons } \\
\mathrm{E}_{\max }=10 \mathrm{MeV}\end{array}$ \\
\hline
\end{tabular}

where inclusion of mixed scattering produces fluxes by an order of magnitude lower than in the case when mixed scattering is neglected. The effect of mixed term is more pronounced in the long-term simulations and for the simulations when waves are assumed to be present at high latitudes and first order resonant scattering dominates at low pitch angles (see section 6). Figure 6 clearly shows that, for a given energy spectrum, the local acceleration is more efficient for Jupiter and can clearly produce the acceleration of electrons on the scale of a few days, while for Saturn the acceleration is much slower. The influence of the initial condition is discussed in more detail in section 6 .

\section{Sensitivity to the Latitudinal Distribution of Waves}

[29] To study how the latitudinal distribution of waves may affect the competition between acceleration and loss on Jupiter and Saturn, we perform calculations with the FDC code but now assuming that waves are present up to $45^{\circ}$ magnetic latitude and not up to $10^{\circ}$ as was assumed in section 3. Figure 7 shows the new diffusion rates.

[30] Since most of electrons at energies below $100 \mathrm{keV}$ resonate with chorus waves within $10^{\circ}-15^{\circ}$ of the geomagnetic environment, extending waves to higher latitudes cannot change the scattering rates for these low-energy particles. At relativistic energies, particles at small equatorial pitch angles will reach these latitudes and will be in resonance with waves. A comparison with Figure 5 shows that extending waves to higher latitudes significantly increases pitch angle scattering close to the edge of the loss cone. Scattering near the loss cone is critical for the loss of electrons to the atmosphere [Roberts, 1969; Shprits et al., 2006c; Albert and Shprits, 2009]. The presence of efficient scattering near the edge of the loss cone may change the balance between local acceleration due to energy diffusion and losses in the loss cone. A similar effect has been studied for the Earth's radiation belts [e.g., Thorne et al., 2005; Shprits et al., 2006c]. Such a large (several orders of magnitude) increase in pitch angle scattering at small equatorial pitch angles results in a dramatic increase in losses.

[31] To quantify the effect of the change of the latitudinal wave distribution, we performed simulations similar to those shown in Figure 6 but with the diffusion coefficients computed assuming that waves extend to $45^{\circ}$ (Figure 7).

[32] The results of the simulations (Figure 8) clearly show that when waves extend to high latitudes the balance between the acceleration and loss can change. In this case, local acceleration is producing a net loss at energies of $1 \mathrm{MeV}$ and below for Saturn and $500 \mathrm{keV}$ and below for Jupiter. There is

\section{Saturn L=6}
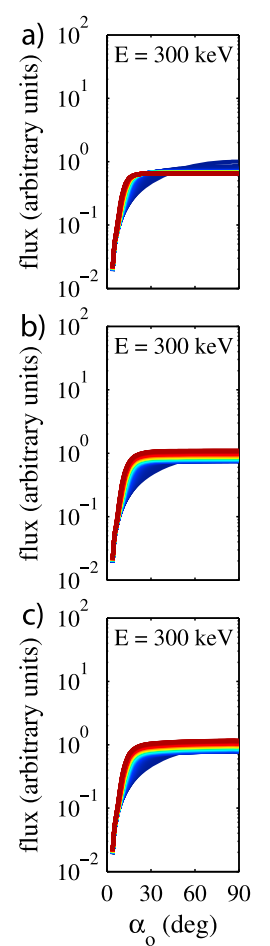
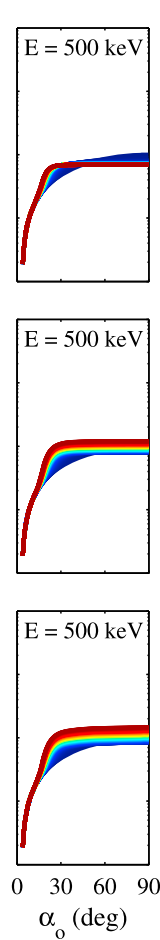
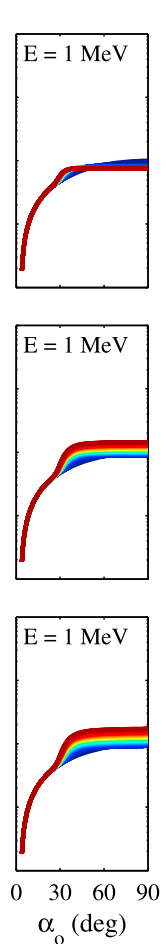
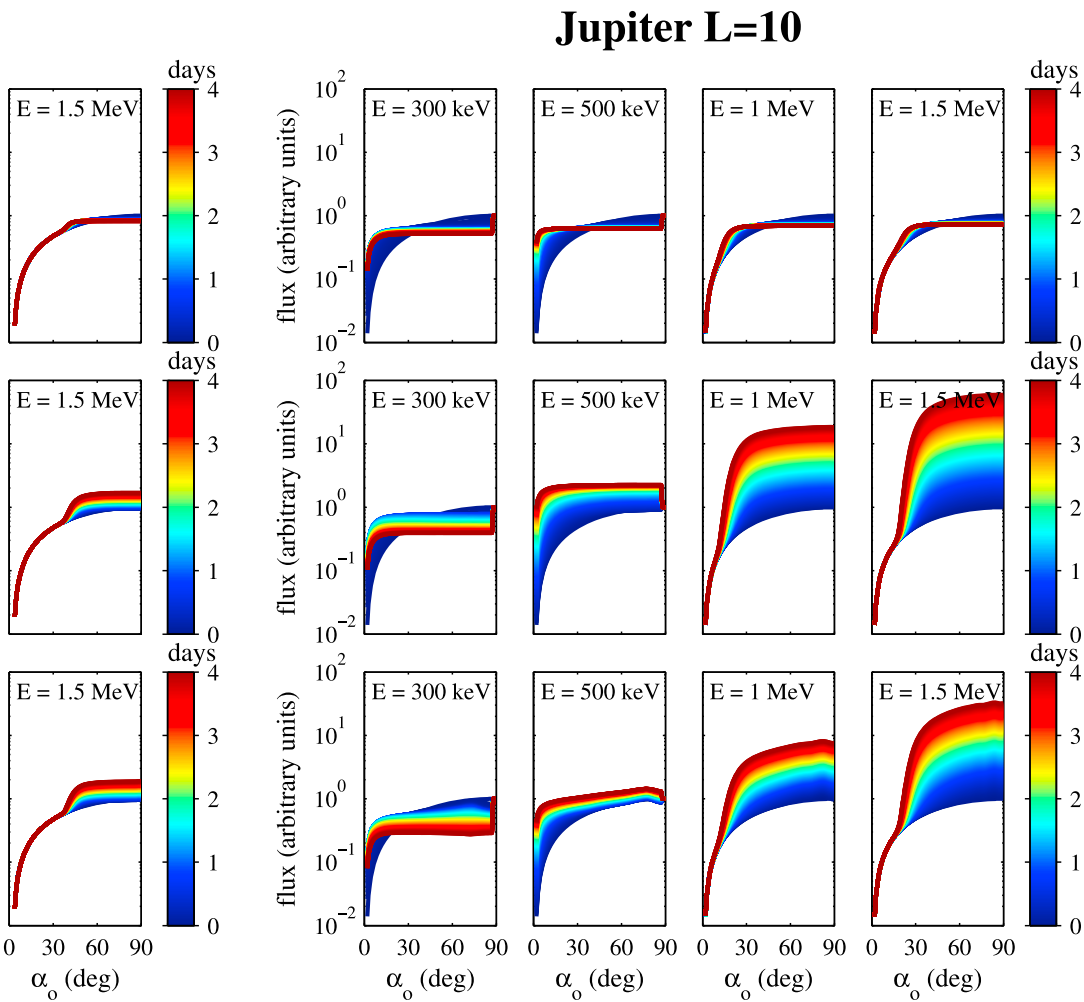

Figure 6 $\alpha_{0}(\operatorname{deg})$
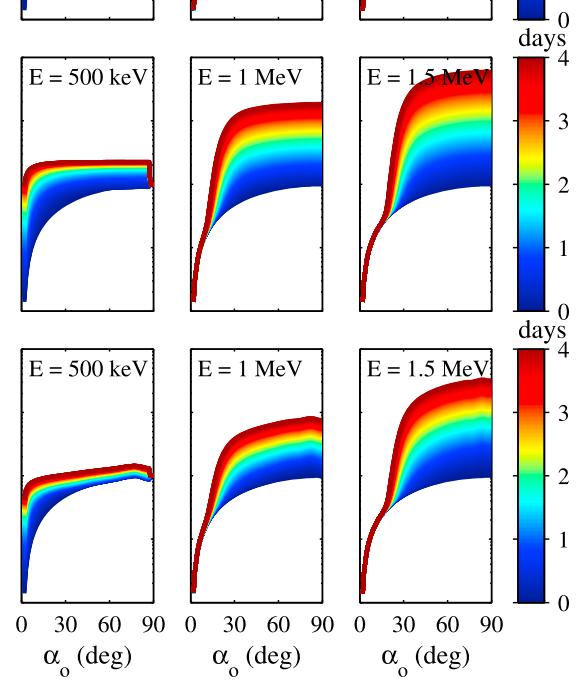
and mixed diffusion for (left) Saturn at $L=6$ and (right) Jupiter at $L=10$. 

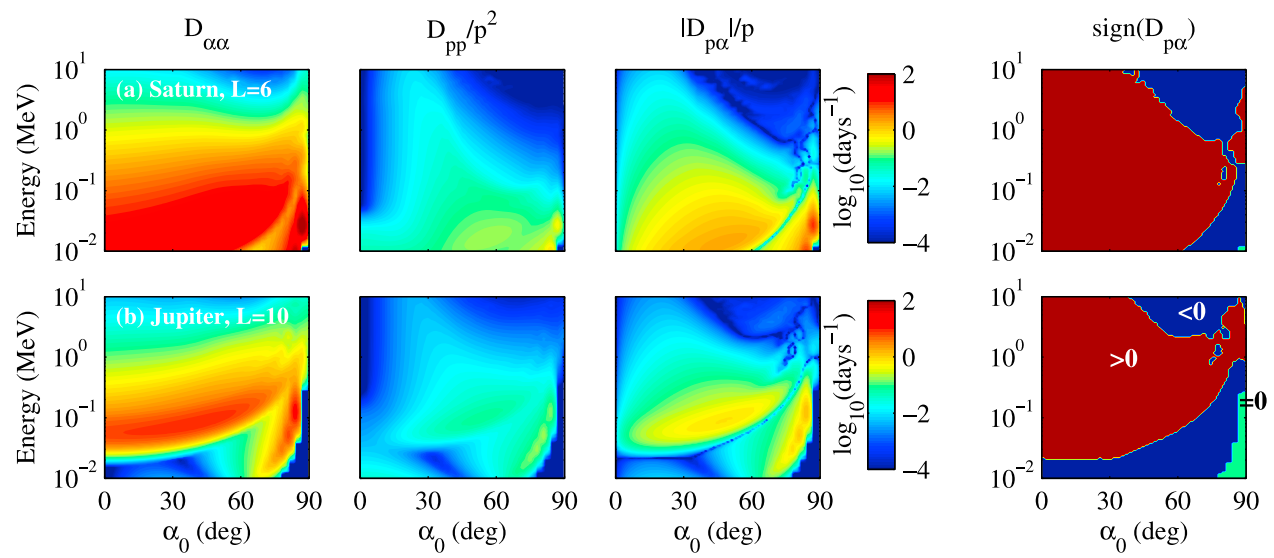

Figure 7. Diffusion rates computed assuming that waves are confined to within $45^{\circ}$ of the magnetic equator for (a) $L=6$ on Saturn and (b) $L=10$ on Jupiter.

still significant acceleration for above $1.5 \mathrm{MeV}$ for Saturn and for greater than $1 \mathrm{MeV}$ for Jupiter.

\section{Sensitivity to the Assumed Initial Distribution}

[33] Energy diffusion depends not only on the scattering rates but also on the gradients in energy spectrum in the initial conditions. To perform more realistic simulations, we introduce initial conditions that are based on observations. If the local acceleration is an important mechanism, the phase space density energy spectrum, derived from observations, may already be affected by the local acceleration. If the local acceleration and loss by chorus waves were the only mechanisms that can violate the adiabatic invariants, a simulation initiated with a realistic energy spectrum simply would not change energy and pitch angle distributions since the initial condition will represent the steady state. If there is an additional loss process, such a simulation with a realistic initial spectrum would produce slow acceleration since this additional loss process was not taken into account.

[34] While a clear interpretation of the results of such simulations is complicated due to the unknown number of

\section{Saturn L=6}

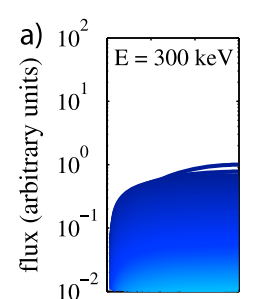

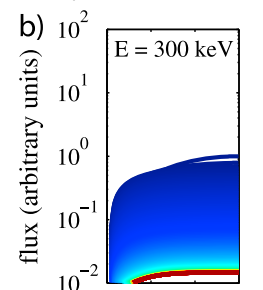

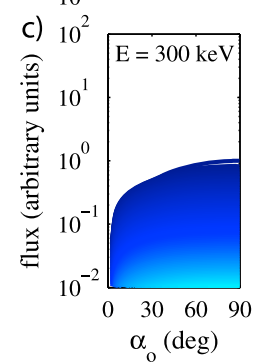

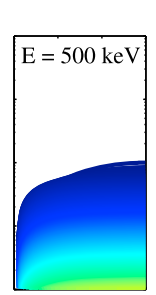
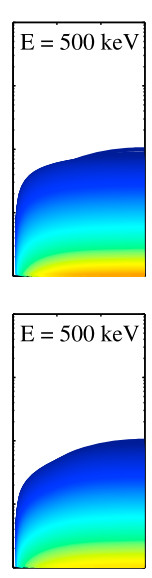

$\alpha_{0}(\operatorname{deg})$
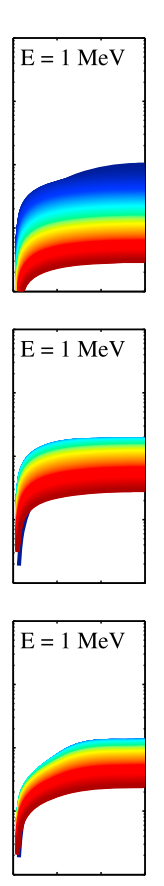

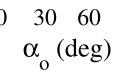
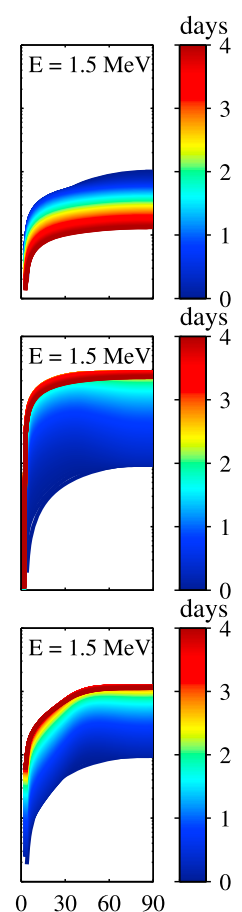

$\alpha_{0}(\operatorname{deg})$
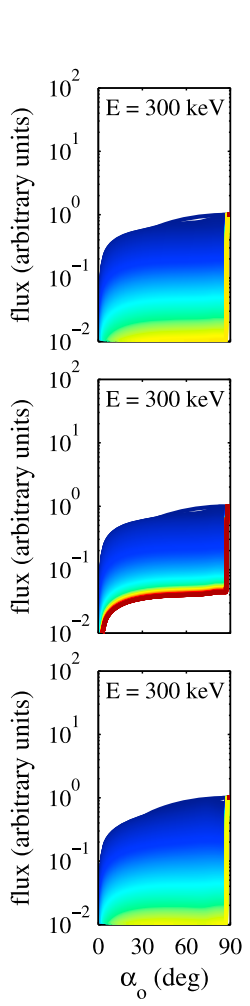

Jupiter $\mathbf{L}=\mathbf{1 0}$
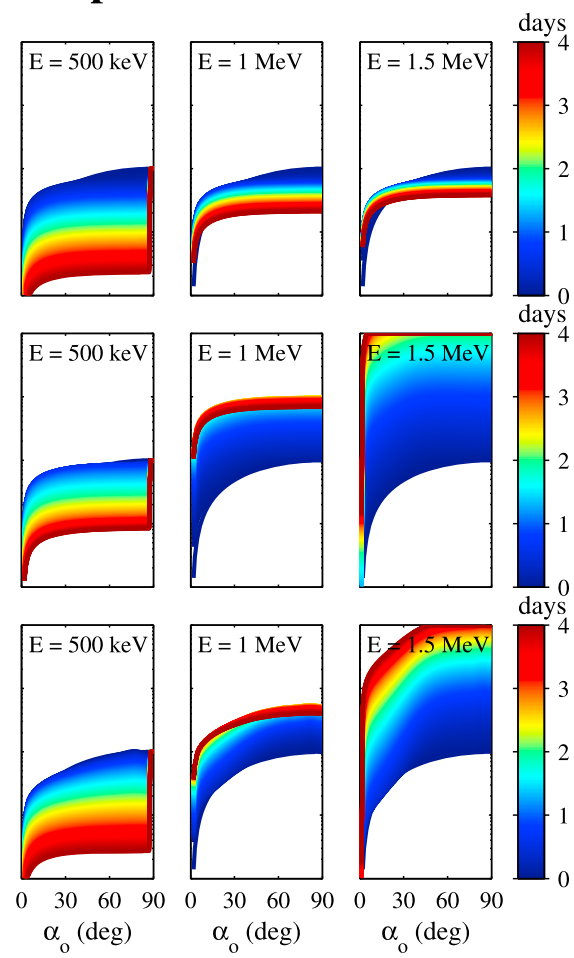

Figure 8. Same as Figure 6 but using diffusion coefficients computed assuming that waves are distributed to $45^{\circ}$ in latitude. (a) Pitch angle diffusion only, (b) pitch angle and energy diffusion, and (c) pitch angle, energy, and mixed diffusion for (left) Saturn at $L=6$ and (right) Jupiter at $L=10$. 


\section{Saturn L=6}
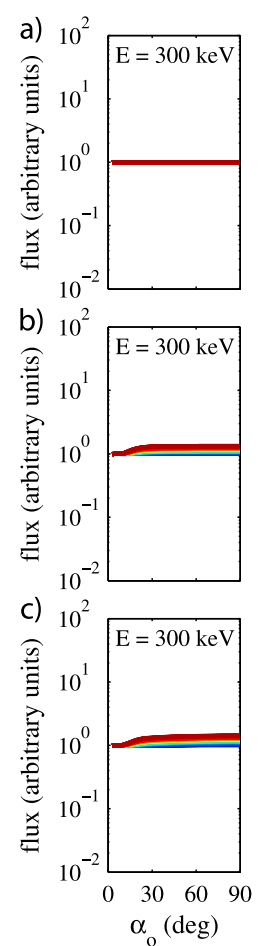
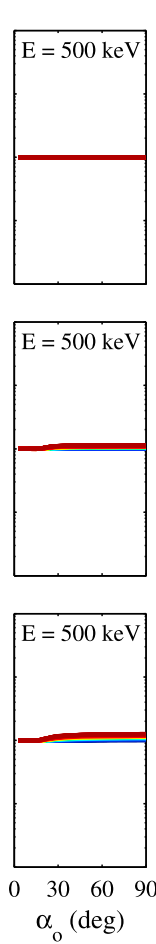
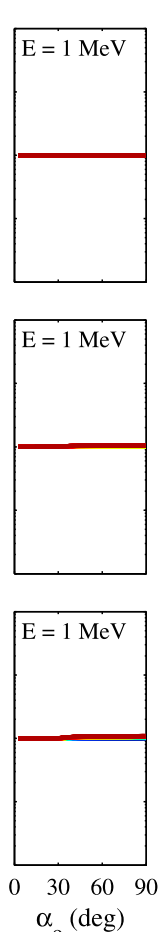
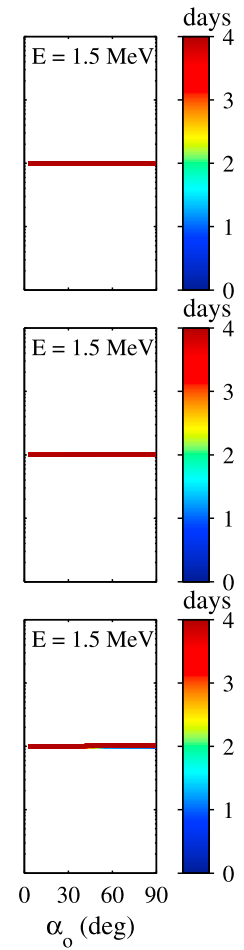

Jupiter $\mathbf{L}=\mathbf{1 0}$
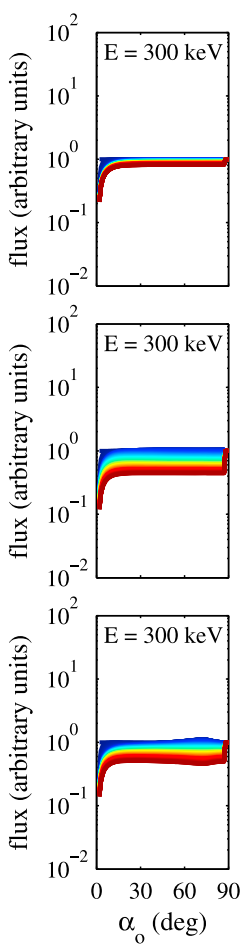
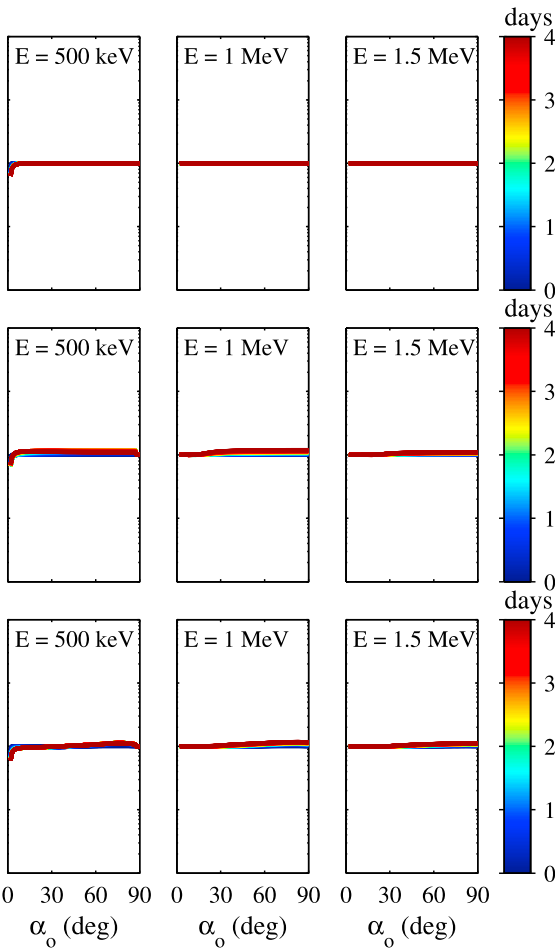

Figure 9. (a) Pitch angle diffusion only, (b) pitch angle and energy diffusion, and (c) pitch angle, energy, and mixed diffusion for (left) Saturn at $L=6$ and (right) Jupiter at $L=10$.

additional source and loss processes, such a simulation is still important as it may help estimating the relative strength of the local acceleration and loss in comparison to other processes. Such simulations can also help study the sensitivity of the simulations to the assumptions of the model. While for waves with wave-normal angles within $45^{\circ}$ the calculated diffusion rates are relatively insensitive to the assumed wavenormal distribution, in the case of highly oblique waves quasi-parallel assumptions will lead to significant errors [Mourenas et al., 2012]. Simulations that include radial diffusion and local acceleration and loss will be a subject of future research. Note that while we start our simulations from the steady state in this section, the electron fluxes will be strongly modulated by the injections that are driven by the interchange instability. Nevertheless the simulations with initial conditions taken from observations can help determine long-term contribution of various acceleration and loss mechanisms and indicate at which energies processes that are not accounted by the 2D model will be required to explain the long-term competition between acceleration and loss in the radiation belts.

[35] The energy spectrum for Saturn at $\mathrm{L}=6$ is taken from Carbary et al. [2011] as

$$
\mathrm{J}(\mathrm{E})=3 \times 10^{4} \times \mathrm{E}^{-1.4}
$$

while for Jupiter we follow Tomás et al. [2004]

$$
\mathrm{J}(\mathrm{E})=10^{6} \times \mathrm{E}^{-1}
$$

where kinetic energy $\mathrm{E}$ is given in $\mathrm{keV}$ and flux is given in $\mathrm{cm}^{-2} \mathrm{sr}^{-1} \mathrm{~s}^{-1} \mathrm{keV}^{-1}$. Note that the measured spectrum for Jupiter is inferred from a relatively small data set obtained on Galileo and is most likely produced by a combination of processes such as injections that are not included in the 2D model. The spectrum for Saturn was obtained using the medial with quartile values in an attempt to exclude the effect of injections but may still be influenced by previous injections. For these simulations we assume that waves are confined to $10^{\circ}$ of the magnetic equator.

[36] The increase in relativistic electrons is much less pronounced in simulations using an energy spectrum inferred from measurements (Figure 9) than for simulations shown on Figure 6. At a few hundred $\mathrm{keV}$, wave-particle interactions actually result in the net loss of electrons. As was discussed above, this result can be expected as the acceleration rates are weaker in the case of a more realistic initial spectrum, which is likely already hardened by the wave-particle interactions. The loss at lower energies is most likely compensated for by the injections driven by the interchange instabilities, which provides a source of electrons similar to the inward radial diffusion in the Earth's radiation belts. While the presented simulations indicate that local acceleration and loss are in balance there are clearly a number of other missing acceleration and loss mechanisms present and operational in the magnetospheres of Jupiter and Saturn.

\section{Summary}

[37] Since plasma frequency to gyrofrequency is one of the main parameters that determine the effectiveness of the 
gyroresonant wave particle interactions, we presented in this study comparative maps of this parameter for Earth, Jupiter, and Saturn. While Earth and Jupiter's environments create preferential conditions for efficient wave-particle interaction of relatively strong magnetic fields, and relatively tenuous plasma, the environment of Saturn has very high plasma densities, which do not allow for efficient wave-particle interactions. Two-dimensional simulations of pitch angle and energy scattering that account for the loss of particles to the dense surface of a planet and for local acceleration due to gyroresonant wave-particles interactions confirm that local acceleration is a potentially very important factor in the acceleration of electrons on Jupiter. While the efficiency of local acceleration and loss depends on the shape of pitch angle and energy distributions produced by other processes, the efficiency of local gyroresonant scattering of electrons by chorus waves is most likely less efficient on Saturn than on Jupiter due to a higher ratio of the plasma frequency to gyrofrequency. Since 2D simulation results are strongly dependent on the initial conditions, 3D modeling including radial diffusion, local acceleration, and pitch angle scattering are required to estimate the relative contribution of each of the acceleration and loss mechanisms. Future work should also account for the stretched magnetic field and diffusion coefficients should be computed in the distorted magnetic field following Orlova and Shprits [2010]. Such modeling should also include the interactions with neutrals, which play an important role at small L-shells for both Jupiter's and Saturn's radiation belt dynamics.

[38] Sensitivity simulations also show that knowledge of the latitudinal distribution of waves is crucially important. If waves extend to high latitudes on Jupiter, the net effect of chorus waves on relativistic electrons will be loss and not acceleration. The high latitude Jovian wave measurements will be provided by the upcoming Juno mission, which will survey wave properties in the inner magnetosphere of Jupiter.

[39] Ineffectiveness of local acceleration on Saturn, presence of losses due to dusty plasmas and losses due to ECH waves that are very strong in Saturn's magnetosphere raises new questions on the other potential acceleration mechanisms that can explain the presence of relativistic electrons in the Kronian environment. Search for these unknown acceleration mechanisms and more accurate quantification of the diffusion processes should be a subject of future research.

[40] Acknowledgments. This study was supported by the NASA grant was primarily supported by NNX11AM36G. It was also supported by the NASA grant NNX09AF51G and NNX10AK996 and by the Lab Fees Research Program.

[41] Masaki Fujimoto thanks the reviewers for their assistance in evaluating this paper.

\section{References}

Albert, J. M. (2005), Evaluation of quasi-linear diffusion coefficients for whistler mode waves in a plasma with arbitrary density ratio, J. Geophys. Res., 110, A03218, doi:10.1029/2004JA010844.

Albert, J. M., and Y. Y. Shprits (2009), Estimates of lifetimes against pitch angle diffusion, J. Atmos. Sol. Terr. Phys., 71, 1647-1652, doi:10.1016/ j.jastp.2008.07.004.

Albert, J. M., and S. L. Young (2005), Multidimensional quasi-linear diffusion of radiation belt electrons, Geophys. Res. Lett., 32, L14110, doi:10.1029/2005GL023191.

Albert, J. M., N. P. Meredith, and R. B. Horne (2009), Three-dimensional diffusion simulation of outer radiation belt electrons during the 9 October
1990 magnetic storm, J. Geophys. Res., 114, A09214, doi:10.1029/ 2009JA014336.

Bagenal, F., and P. A. Delamere (2011), Flow of mass and energy in the magnetospheres of Jupiter and Saturn, J. Geophys. Res., 116, A05209, doi:10.1029/2010JA016294

Baker, D. N. (2002), How to cope with space weather, Science, 297(5586), 1486-1487, doi:10.1126/science.1074956.

Bolton, S. J., R. M. Thorne, D. A. Gurnett, W. S. Kurth, and D. J. Williams (1997), Enhanced whistler-mode emissions: Signatures of interchangemotion in the Io torus, Geophys. Res. Lett., 24, 2123-2126, doi:10.1029/ 97GL02020.

Bolton, S. J., et al. (2002), Ultra-relativistic electrons in Jupiter's radiation belts, Nature, 415, 987-991, doi:10.1038/415987a.

Branson, N. J. B. A. (1968), High resolution observations of the planet, Monthly Notices R. Astron. Soc., 139, 155-162.

Brice, N., and T. R. McDonough (1973), Jupiter's radiation belts, Icarus, 18(2), 206-219, doi:10.1016/0019-1035(73)90204-2.

Bunch, N. L., M. Spasojevic, and Y. Y. Shprits (2011), On the latitudinal extent of chorus emissions as observed by the Polar Plasma Wave Instrument, J. Geophys. Res., 116, A04204, doi:10.1029/2010JA016181.

Bunch, N. L., M. Spasojevic, and Y. Y. Shprits (2012), Off-equatorial chorus occurrence and wave amplitude distributions as observed by the Polar Plasma Wave Instrument, J. Geophys. Res., 117, A04205, doi:10.1029/2011JA017228.

Carbary, J. F., C. Paranicas, D. G. Mitchell, S. M. Krimigis, and N. Krupp (2011), Energetic electron spectra in Saturn's plasma sheet, J. Geophys. Res., 116, A07210, doi:10.1029/2011JA016598.

Carr, T. D., and S. Gulkis (1969), The magnetosphere of Jupiter, Annu. Rev. Astron. Astrophys., 7, 577-618, doi:10.1146/annurev.aa.07.090169.003045.

Cohen, C. M. S., E. C. Stone, and R. S. Selesnick (2001), Energetic ion observations in the middle Jovian magnetosphere, J. Geophys. Res., 106, 29,871-29,881, doi:10.1029/2001JA000008.

Drake, F. D., and S. Hvatum (1959), Non-thermal microwave radiation, Astron. J., 64(8), 329-330, doi:10.1086/108047.

Fälthammar, C.-G. (1965), Effects of time-dependent electric fields on geomagnetically trapped radiation, J. Geophys. Res., 70, 2503-2516, doi:10.1029/JZ070i011p02503.

Frank, L. A., W. R. Paterson, and K. K. Khurana (2002), Observations of thermal plasmas in Jupiter's magnetotail, J. Geophys. Res., 107(A1), 1003, doi:10.1029/2001JA000077.

Glauert, S. A., and R. B. Horne (2005), Calculation of pitch angle and energy diffusion coefficients with the PADIE code, J. Geophys. Res., 110, A04206, doi:10.1029/2004JA010851.

Gurnett, D. A., W. S. Kurth, and F. L. Scarf (1981), Plasma waves near Saturn: Initial results from Voyager 1, Science, 212(4491), 235-239, doi:10.1126/science.212.4491.235.

Horne, R. B., and R. M. Thorne (1998), Potential wave modes for electron scattering and stochastic acceleration to relativistic energies during magnetic storms, Geophys. Res. Lett., 25, 3011, doi:10.1029/98GL01002.

Horne, R. B., et al. (2005a), A critical test of electron acceleration in the Van Allen radiation belts, Nature, 437(8), 227-230, doi:10.1038/ nature03939.

Horne, R. B., R. M. Thorne, S. A. Glauert, J. M. Albert, N. P. Meredith, and R. R. Anderson (2005b), Timescale for radiation belt electron acceleration by whistler mode chorus waves, J. Geophys. Res., 110, A03225, doi:10.1029/2004JA010811.

Horne, R. B., R. M. Thorne, S. A. Glauert, J. D. Menietti, Y. Y. Shprits, and D. A. Gurnett (2008), Gyro-resonant electron acceleration inside Jupiter's radiation belt, Nat. Phys., 4(4), 301-304, doi:10.1038/nphys897.

Hospodarsky, G. B., T. F. Averkamp, W. S. Kurth, D. A. Gurnett, J. D. Menietti, O. Santolik, and M. K. Dougherty (2008), Observations of chorus at Saturn using the Cassini Radio and Plasma Wave Science instrument, J. Geophys. Res., 113, A12206, doi:10.1029/2008JA013237.

Katoh, Y., F. Tsuchiya, Y. Miyoshi, A. Morioka, H. Misawa, R. Ujiie, W. S. Kurth, A. T. Tomás, and N. Krupp (2011), Whistler mode chorus enhancements in association with energetic electron signatures in the Jovian magnetosphere, J. Geophys. Res., 116, A02215, doi:10.1029/2010JA016183.

Kellogg, P. J. (1959), Van Allen radiation of solar origin, Nature, 183, 1295-1297, doi:10.1038/1831295a0.

Kivelson, M. G., K. K. Khurana, C. T. Russell, and R. J. Walker (1997), Intermittent short-duration magnetic field anomalies in the Io torus: Evidence for plasma interchange?, Geophys. Res. Lett., 24, 2127-2130, doi:10.1029/97GL02202.

Koons, H. C., and J. L. Roeder (1990), A survey of equatorial magnetospheric wave activity between 5 and $8 \mathrm{R}_{\mathrm{E}}$, Planet. Space Sci., 38(10), 1335-1341, doi:10.1016/0032-0633(90)90136-E

Lenchek, A. M., S. F. Singer, and R. C. Wentworth (1961), Geomagnetically trapped electrons from cosmic ray albedo neutrons, J. Geophys. Res., 66(12), 4027-4046, doi:10.1029/JZ066i012p04027. 
Li, W., Y. Y. Shprits, and R. M. Thorne (2007), Dynamic evolution of energetic outer zone electrons due to wave-particle interactions during storms, J. Geophys. Res., 112, A10220, doi:10.1029/2007JA012368.

Liu, X., T. W. Hill, R. A. Wolf, S. Sazykin, R. W. Spiro, and H. Wu (2010), Numerical simulation of plasma transport in Saturn's inner magnetosphere using the Rice Convection Model, J. Geophys. Res., 115, A12254, doi:10.1029/2010JA015859.

Lorenzato, L., A. Sicard, and S. Bourdarie (2012), A physical model for electron radiation belts of Saturn, J. Geophys. Res., 117, A08214, doi:10.1029/2012JA017560.

McKibben, R. B., and J. A. Simpson (1980), Charged particle diffusion and acceleration in Saturn's radiation belts, J. Geophys. Res., 85, 5773-5783, doi:10.1029/JA085iA11p05773.

Meredith, N. P., R. B. Horne, R. M. Thorne, and R. R. Anderson (2003), Favored regions for chorus-driven electron acceleration to relativistic energies in the Earth's outer radiation belt, Geophys. Res. Lett., 30(16), 1871, doi:10.1029/2003GL017698.

Miyoshi, Y., A. Morioka, H. Misawa, T. Obara, T. Nagai, and Y. Kasahara (2003), Rebuilding process of the outer radiation belt during the 3 November 1993 magnetic storm: NOAA and Exos-D observations, J. Geophys. Res., 108(A1), 1004, doi:10.1029/2001JA007542.

Mourenas, D., A. V. Artemyev, J.-F. Ripoll, O. V. Agapitov, and V. V. Krasnoselskikh (2012), Timescales for electron quasi-linear diffusion by parallel and oblique lower-band chorus waves, J. Geophys. Res., 117, A06234, doi:10.1029/2012JA017717.

Ni, B., R. M. Thorne, Y. Y. Shprits, and J. Bortnik (2008), Resonant scattering of plasma sheet electrons by whistler-mode chorus: Contribution to diffuse auroral precipitation, Geophys. Res. Lett., 35, L11106, doi:10.1029/2008GL034032.

Orlova, K. G., and Y. Shprits (2010), Dependence of pitch-angle scattering rates and loss timescales on the magnetic field model, Geophys. Res. Lett., 37, L05105, doi:10.1029/2009GL041639.

Persoon, A. M., D. A. Gurnett, W. S. Kurth, and J. B. Groene (2006), A simple scale height model of the electron density in Saturn's plasma disk, Geophys. Res. Lett., 33, L18106, doi:10.1029/2006GL027090.

Radhakrishnan, V., and J. A. Roberts (1960), Polarization and Angular Extent of the $960-\mathrm{Mc} / \mathrm{sec}$ Radiation from Jupiter, Phys. Rev. Lett., 4, 493-494, doi:10.1103/PhysRevLett.4.493.

Roberts, C. S. (1969), Pitch-angle diffusion of electrons in the magnetosphere, Rev. Geophys., 7, 305-337, doi:10.1029/RG007i001p00305.

Rymer, A. M., B. H. Mauk, T. W. Hill, C. Paranicas, D. G. Mitchell, A. J. Coates, and D. T. Young (2008), Electron circulation in Saturn's magnetosphere, J. Geophys. Res., 113, A01201, doi:10.1029/2007JA012589.

Santos-Costa, D., and S. A. Bourdarie (2001), Modeling the inner Jovian electron radiation belt including non-equatorial particles, Planet. Space Sci., 49, 303-312, doi:10.1016/S0032-0633(00)00151-3.

Santos-Costa, D., M. Blanc, S. Maurice, and S. J. Bolton (2003), Modeling the electron and proton radiation belts of Saturn, Geophys. Res. Lett., 30(20), 2059, doi:10.1029/2003GL017972.

Santos-Costa, D., S. J. Bolton, R. M. Thorne, Y. Miyoshi, and S. M. Levin (2008), Investigating the origins of the Jovian decimetric emission's variability, J. Geophys. Res., 113, A01204, doi:10.1029/2007JA012396.

Scarf, F. L., and D. A. Gurnett (1977), A plasma wave investigation for the Voyager Mission, Space Sci. Rev., 21(3), 289-308, doi:10.1007/ BF00211543.

Scarf, F. L., D. A. Gurnett, W. S. Kurth, and R. L. Poynter (1982), Voyager2 plasma-wave observations at Saturn, Science, 215(4532), 587-594, doi:10.1126/science.215.4532.587.

Schulz, M., and L. J. Lanzerotti (1974), Particle Diffusion in the RadiationBelts, Phys. Chem. Space, vol. 7, 215 pp., Springer, New York.

Sheeley, B. W., M. B. Moldwin, H. K. Rassoul, and R. R. Anderson (2001), An empirical plasmasphere and trough density model: CRRES observations, J. Geophys. Res., 106, 25,631-25,641, doi:10.1029/2000JA000286.

Shprits, Y. Y., and B. Ni (2009), Dependence of the quasi-linear scattering rates on the wave normal distribution of chorus waves, J. Geophys. Res., 114, A11205, doi:10.1029/2009JA014223.

Shprits, Y. Y., and R. M. Thorne (2004), Time dependent radial diffusion modeling of relativistic electrons with realistic loss rates, Geophys. Res. Lett., 31, L08805, doi:10.1029/2004GL019591.

Shprits, Y. Y., R. M. Thorne, R. B. Horne, S. A. Glauert, M. Cartwright, C. T. Russell, D. N. Baker, and S. G. Kanekal (2006a), Acceleration mechanism responsible for the formation of the new radiation belt during the 2003 Halloween solar storm, Geophys. Res. Lett., 33, L05104, doi:10.1029/2005GL024256.

Shprits, Y. Y., R. M. Thorne, R. Friedel, G. D. Reeves, J. Fennell, D. N. Baker, and S. G. Kanekal (2006b), Outward radial diffusion driven by losses at magnetopause, J. Geophys. Res., 111, A11214, doi:10.1029/ 2006JA011657.
Shprits, Y. Y., W. Li, and R. M. Thorne (2006c), Controlling effect of the pitch angle scattering rates near the edge of the loss cone on electron lifetimes, J. Geophys. Res., 111, A12206, doi:10.1029/2006JA011758.

Shprits, Y. Y., R. M. Thorne, R. B. Horne, and D. Summers (2006d), Bounce-averaged diffusion coefficients for field-aligned chorus waves, J. Geophys. Res., 111, A10225, doi:10.1029/2006JA011725.

Shprits, Y. Y., N. P. Meredith, and R. M. Thorne (2007), Parameterization of radiation belt electron loss timescales due to interactions with chorus waves, Geophys. Res. Lett., 34, L11110, doi:10.1029/2006GL029050.

Shprits, Y. Y., D. A. Subbotin, N. P. Meredith, and S. R. Elkington (2008), Review of modeling of losses and sources of relativistic electrons in the outer radiation belts: II. Local acceleration and loss, J. Atmos. Sol. Terr. Phys., 70(14), 1694-1713, doi:10.1016/j.jastp.2008.06.014.

Shprits, Y. Y., D. Subbotin, and B. Ni (2009), Evolution of electron fluxes in the outer radiation belt computed with the VERB code, J. Geophys. Res., 114, A11209, doi:10.1029/2008JA013784.

Sicard, A., and S. Bourdarie (2004), Physical electron belt model from Jupiter's surface to the orbit of Europa, J. Geophys. Res., 109, A02216, doi:10.1029/2003JA010203.

Sicard, A., et al. (2004), Long-term dynamics of the inner Jovian electron radiation belts, Adv. Space Res., 33, 2039-2044, doi:10.1016/j.asr.2003. 04.053.

Simpson, J. A., T. S. Bastian, D. L. Chenette, R. B. McKibben, and K. R. Pyle (1980), The trapped radiations of Saturn and their absorption by satellites and rings, J. Geophys. Res., 85, 5731-5762, doi:10.1029/ JA085iA1 1 p05731.

Siscoe, G. L., and D. Summers (1981), Centrifugally driven diffusion of iogenic plasma, J. Geophys. Res., 86, 8471-8479, doi:10.1029/ JA086iA10p08471.

Stannard, D., and R. G. Conway (1976), Recent observations of decametric radio-emission from Jupiter, Icarus, 27(3), 447-452, doi:10.1016/00191035(76)90023-3

Su, Z., F. Xiao, H. Zheng, and S. Wang (2010), STEERB: A threedimensional code for storm-time evolution of electron radiation belt, J. Geophys. Res., 115, A09208, doi:10.1029/2009JA015210.

Subbotin, D. A., and Y. Y. Shprits (2009), Three-dimensional modeling of the radiation belts using the Versatile Electron Radiation Belt (VERB) code, Space Weather, 7, S10001, doi:10.1029/2008SW000452.

Subbotin, D. A., et al. (2011), Simulation of the acceleration of relativistic electrons in the inner magnetosphere using RCM-VERB coupled codes, J. Geophys. Res., 116, A08211, doi:10.1029/2010JA016350.

Summers, D., R. M. Thorne, and F. Xiao (1998), Relativistic theory of waveparticle resonant diffusion with application to electron acceleration in the magnetosphere, J. Geophys. Res., 103, 20,487-20,500, doi:10.1029/ 98JA01740.

Tao, X., J. M. Albert, and A. A. Chan (2009), Numerical modeling of multidimensional diffusion in the radiation belts using layer methods, J Geophys, Res, 114, A02215, doi:10.1029/2008JA013826.

Thomsen, M. F., D. B. Reisenfeld, D. M. Delapp, R. L. Tokar, D. T. Young, F. J. Crary, E. C. Sittler, M. A. McGraw, and J. D. Williams (2010), Survey of ion plasma parameters in Saturn's magnetosphere, J. Geophys. Res., 115, A10220, doi:10.1029/2010JA015267.

Thorne, R. M., T. P. Armstrong, S. Stone, D. J. Williams, R. W. McEntire, S. J. Bolton, D. A. Gurnett, and M. G. Kivelson (1997), Galileo evidence for rapid interchange transport in the Io torus, Geophys. Res. Lett., 24, 2131-2134, doi:10.1029/97GL01788.

Thorne, R. M., T. P. O'Brien, Y. Y. Shprits, D. Summers, and R. B. Horne (2005), Timescale for MeV electron microburst loss during geomagnetic storms, J. Geophys. Res., 110, A09202, doi:10.1029/2004JA010882.

Tomás, A. T., J. Woch, N. Krupp, A. Lagg, K.-H. Glassmeier, and W. S. Kurth (2004), Energetic electrons in the inner part of the Jovian magnetosphere and their relation to auroral emissions, J. Geophys. Res., 109, A06203, doi:10.1029/2004JA010405.

Tsurutani, B. T., and E. J. Smith (1977), Two types of magnetospheric ELF chorus and their substorm dependences, J. Geophys. Res., 82, 5112-5128, doi:10.1029/JA082i032p05112.

Tverskoy, B. A. (1965), Transport and acceleration of charged particles in the earth's magnetosphere, Geomagn. Aeron., Engl. Transl., 5(5), 793-808.

Van Allen, J. A., M. F. Thomsen, B. A. Randall, R. L. Rairden, and C. L. Grosskreutz (1980), Saturn's magnetosphere, rings and inner satellites, Science, 207(4429), 415-421, doi:10.1126/science.207.4429.415.

Varotsou, A., D. Boscher, S. Bourdarie, R. B. Horne, S. A. Glauert, and N. P. Meredith (2005), Simulation of the outer radiation belt electrons near geosynchronous orbit including both radial diffusion and resonant interaction with Whistler-mode chorus waves, Geophys. Res. Lett., 32, L19106, doi:10.1029/2005GL023282. 